\title{
Validity of Psychotherapy in Treating Chinese Adolescents with Anorexia Nervosa
}

\author{
Xiaozhi Luo ${ }^{1, *}$ \\ ${ }^{1}$ University of Bradford, City of Bradford, England \\ ${ }^{*}$ Corresponding author. Email: guanghua.ren@gecacademy.cn
}

\begin{abstract}
The essay is written to educate the general public about Anorexia and its corresponding treatments. It dwells on the etiology and impacts of Anorexia Nervosa. The application and effectiveness of Cognitive Behavioral Therapy and Dialectical Behavioral Therapy will be interpreted later, emphasizing the cultural contexts, risk factors, and impacts of this specific eating disorder among Chinese adolescents. After that, the conclusion and limitations of the current study of these two therapies. The expectation of its future development will also be presented at the end of the dissertation. This essay faces people interested in Anorexia Nervosa and those who keep following eyes on the application of mental therapies for eating problems.
\end{abstract}

Keywords: Eating disorders, Chinese adolescent, Anorexia Nervosa, Psychological therapies

\section{INTRODUCTION}

\subsection{Anorexia Nervosa}

According to DSM-5, the diagnostic criteria of Anorexia Nervosa include restriction of energy intake relative to requirements that contributes to abnormal body weight and will have negative effects on libido, physical health, and social relationships, etc. Also, the patient is likely to be intensely concerned with being fat even though at a significantly low weight. They persistently lack correct recognition of the seriousness of their body image or weight [1]. The etiology for Anorexia Nervosa is yet inconclusive. Still, there are a few potential risk factors for this life-threatening illness, like past criticism about their weight, figure, or eating habits, low self-esteem, a personality that tends toward perfectionism, or history of bullying about body [2]. Comorbidities of AN include transition to yellow-orange dry skin, bone fractures, cardiovascular problems, etc. [3].

\subsection{Adolescents in China}

Nowadays, adolescents in China face great expectations on their figures from society, which brings them extremely high pressure than ever. People appreciate "BaiYouShou", a modern esthetic standard that basically stands for pale skin, younger look, and thin figure that sometimes turns out to be abnormal, unhealthy, and obviously lack of nutrition. Strict esthetic standards like this setting for people really lead to incorrect body images and further anxiety about their weight or looks.

Plus, most adolescents in China don't really have a clear picture of how they're supposed to look but unconsciously follow the rules resulting from the trend, which blind them and make conditions worse. They have been relying their behaviors and ideas too much on how other people comment or criticize instead of how they think of themselves.

\subsection{Treatments}

Considering some cases with intense family relationships, we concern that Family Therapy might not be proper for people who have a traditional Chinese family. Modern adolescents sometimes do not get along well with their parents or siblings, making it a barrier to apply Family Therapy since they gain too much pressure from such a vibe. Also, some parents hold conservative ideas about mental illnesses that block the way their children receive proper treatment. In conclusion, this is basically why we recommend CBT or DBT as a treatment for AN instead of Family Therapy. Studies have shown that CBT and DBT in combination with pharmaceutical therapies both 
reduce the recurrence rate and provide patients with a longer term, stable mental health [4].

Thus, the purpose of this article is to educate the general public about symptoms, criteria, prevalence, risk factors, and comorbidities of $\mathrm{AN}$, also to compare the effectiveness of CBT and DBT as its corresponding treatments through literature from past and present and relevant experiments.

\section{LITERATURE}

\subsection{Etiology}

This part basically introduces risk factors and impacts of Anorexia Nervosa, which are crucial when discussing the application and the effectiveness of CBT and DBT

\subsubsection{Biological Risk Factors}

Risk factors of Anorexia Nervosa can be different and variable within contexts. The listed factors, such as family history (genetics), might contribute to eating disorders. When the subject has siblings or parents who have had eating disorders in the past, he/she is more likely to get one. From the perspective of dieting to another extent, starvation might lead to changes in mood, rigidity in thinking processes, and reduction in appetite, which is likely to be prolonged. It changes the way the brain functions within certain sections so a vulnerable individual might find it hard to return to normal diets again [5].

In addition, the family environment shall be crucial, for example. Young people that turn out to grow under a stressful family that offer a lack of love and respect might take not eating as a way to exercise their power since they are not likely to in other aspects of life. People sometimes refuse to eat due to extreme stress or insecurity, which is normal when facing family problems or seeing intense family conflicts themselves [6].

An imbalance in brain chemicals leads to eating disorders too. Since it is similar to how people get depression, it is possible to assume that people who suffer from eating disorders might also suffer from depression and other mental illnesses caused by the imbalance of chemicals. Specific examples of chemical messengers in the brain that can become imbalanced in eating disorders and in mood disorders include cortisol, which is involved in the stress response, and dopamine and tryptophan, which can help us relax. As it goes, AN leads to a worse and worse situation of the patient's health condition since fat and energy are needed to produce certain chemicals, which makes it more difficult for the patient to get better and recover [7].

\subsubsection{Psychological Risk Factors}

The main course of this specific eating disorder is generally associated with stressful life events as well. It might be a contributing factor that increases the possibility of getting anorexia nervosa. It is generally induced by certain incidents such as moving to a new place or facing a seemingly unsolvable crisis. In this case, younger individuals are likely to display abnormal features such as denying fear of being fat [8].

Social pressure, to the psychological extent, mainly takes place through social media and other online forms of entertainment, which will have a negative impact that causes anorexia nervosa since it possibly leads the patient to an incorrect body image. Unfriendly, negative comments posted online will definitely impact the mental condition of the subject and create an excessively improper perspective of how they look, later contributing to abnormal eating habits since they want their shapes and figures to be nicely commented on by others instead of being denied. Also, how society sets the standard for beauty counts the most, so does the extent of resistance of the subject when facing such pressure. It is also why women are more likely to have eating disorders than men. Women are pressured by society to be thin, leading to eating disorders, while men are pressured by society to be large. With the development of social media and networks, an influx of information created through movies, dramas, and online journals forces us to be thinner and thinner to meet the standard [6]. It is reasonable that the media tends to make more fit actors and actresses the heroes and loads of films and television programs, but sometimes negative impacts exceed the positive, making audiences more and more anxious about their figures and appearances while they did not even notice. It is exactly the same case that the companies want to sell their products as many as possible in marketing. They have to make advertisements by inviting fashion models that are generally underweight. It is reasonable, but it really makes people anxious that even people of a healthy weight can see themselves as not living up to imaginary or impossible expectations.

\subsection{Impact}

\subsubsection{Social Life and Individual Mental Health}

Just like other mental illnesses, anorexia has its complications both to a physical and psychological extent. While some individuals remain active in social functioning as always, some might undergo a significant state of isolation or a sense of failure in his/her academic or professional careers. The patients with AN are often reported a sense of loneliness, 
abandonment, and isolation. They have reduced social capital since they might lose friends in the process of treatment. Many patients claim that due to their prolonged illness and that they must stay in hospital for most of the time, they likely don't get along with their friends or spend time together. When their friends ask whether they'd get out soon or when they'd get better, the patients can't really give a date or anything, which might make them feel helpless and depressed. Patients frequently reported feeling that life in hospital was static and life on the outside for friends was dynamic, with long periods of absence affecting their friendships. And when there is a long distance from where they originally lived to the hospital they stay at present, it might be hard for these patients to maintain old relationships due to a lack of contact, and they are not likely to establish new ones at all. Under this situation, they tend to feel abandoned by their friends, and it may lead to chronic distress later if it has been a prolonged period [9]. Besides, it has been found that AN can complicate or compromise intimacy-which is crucial in a relationship. Since patients with AN are likely to experience low self-esteem and incorrect perception of their body image, they will reduce libido when food restriction also decreases hormonal functioning, which plays an important role in sexual activities. It will thus have an impact on the intimacy of the partners [10].

\subsubsection{Impact on Physical Health}

Anorexia nervosa influences the patients in ways more than mental health. Over $90 \%$ of AN patients develop secondary amenorrhea due to chronic malnutrition. Amenorrhea results from a decrease in FSH and corpus luteum hormone. Also, among AN's complications, the reduction of bone mass is a serious problem as it is difficult to cure. Bone mass is reduced by $40 \%$ to $60 \%$ of other adolescents. This could be dangerous for the patients later in life since a reduction in bone mass undoubtedly leads to a higher risk of bone fracture than others do [11]. According to the data, It is reported that mitral valve prolapse is seen in $32 \%$ to $60 \%$ of AN patients, in comparison to $6 \%$ to $22 \%$ of the general population. The patients will have a higher risk to have of heart failure, especially after 2 weeks that they get nutrient supplementations, which leads to an increase in the blood that circulates since there's a sudden supplement of nutrients. Plus, an increase in hydropericardium might also be higher in the risk of heart failure.

\subsection{Treatments}

\subsection{1. $C B T$}

Cognitive behavioral therapy (CBT) as an outpatient intervention for anorexia nervosa after hospitalization is designed to prevent patients from relapses after weight gain during hospitalization. CBT for acute weight gain aims to restore weight on an outpatient basis. CBT for anorexia nervosa is designed for late teens and adults with the disorder and is typically administered to individuals over a period of one year. This treatment specifically focuses on helping the patients to get back to their normal state of a healthy weight or figure. It uses behavioral strategies that might include establishing regular eating patterns and systematic exposure to prohibited foods. CBT also emphasizes schema level change, challenging the seemingly inextricable link between identity and the illness. There are three main stages of CBT: the behavioral stage, cognitive stage, maintenance stage, and relapse stage. CBT is usually used in combination with medication or clinical therapy, depending on the patient. Studies have shown that CBT reduces the recurrence rate and provides patients with the longer term, stable mental health [4]. According to the data in previous research, after 1 year, $65 \%$ of patients receiving CBT had not relapsed, and $34 \%$ of the group receiving "treatment as usual" had not relapsed. These findings suggest that CBT may be more effective than patients not receiving regular individual psychotherapy in preventing relapse.

\subsection{2. $D B T$}

\subsubsection{Development}

Dialectical behavior therapy is a philosophy-based therapy, which serves structured intervention performed by therapists trained psychological principles designed to help the person change their way of thinking, behaving for adjusting negative insight when uncertainty comes to their life. According to the recovery first treatment center, DBT is a complicated type of cognitive behavior that developed from CBT therapy and was often used to treat individuals with severe problems. Unfortunately, the integration problems found in cultures, beliefs, and perspectives created huge barriers in the treatment process made the requirement of the full set of training complex to master before therapists apply the principles of DBT [12].

DBT was first used in the treatment of BPD. After that, DBT was improved and combined with other therapists for various psychological disorders, such as eating disorders, substance abuse, serve depression, and those with intellectual disabilities [13]. Today, DBT has been adapted both as a primary treatment intervention and supplemental treatment for adolescents. Countless articles presenting efficacy data have consistently shown great promises of adapting DBT principles in treating clients with binge eating, such as DBT-BED, DBT-BN, DBT-FBT, and other 
combined treatments. A study designed in 2001 suggested that 89 percent of participants were symptom-free when the experiment concluded, while 56 percent remained symptom-free in the six-months follow-up. The results also showed an association between DBT and increased BMI. A recent study on the use of dialectical behavioral therapy, with a small sample of adults that being diagnosed to have anorexia nervosa involved, has suggested a strong relationship between reduction in symptoms and DBT [14]. As the most resistant type of ED to treatment, Anorexia nervosa has been given less attention by DBT therapists. The availability of DBT on this specific type of Eating disorder has been questioned as a consequence. In 2016, Behavioral Tech concluded the current studies and pointed out they primarily included individuals presenting with low, moderate levels of ED symptoms and excluded those with Anorexia nervosa and multiple treatment targets [15].

In recent years, much inspiring progress if DBT's effective use in Anorexia nervosa has been made, and new variations of DBT are also being explored, such as Radically open dialectical behavior therapy (RO DBT).

\subsubsection{2. $R O D B T$}

Current studies discovered certain validity of DBT in AN treatment. Focusing on relieving the association of excessive self-control, which is commonly seen in AN patient's consciousness, experiments were set for on passing skills of addressing emotional and behavior over-control to Adult AN for reducing rational sensitivity and promoting response system toward punishing imperfection. Case series 1 adopted the classic DBT, resulting in the retention of around $83 \%$ of recovery rate and 0.5 progress in BMI. Case series 2 combined standard DBT with skills of managing over-control ideas. Around $89 \%$ of participants maintain the effect size increase in BMI in the following 6- and 12-months follow-up [16]. Although therapists earn positive results, further study is still necessary for this model for AN.

From evolutionary standpoints, RO DBT values emotion management in communication and connection [17]. According to Lynch, Maladaptive over control could be one of the features that Anorexia Nervosa embodied, including rigid and rule governed behaviors, extreme inhibition of needs, and a high level of perfectionism [18]. Individuals are expected to find the method of dealing with social events properly after treatment.

In an uncontrolled trial with women diagnosed with anorexia nervosa, Lynch and peers found that after an average of 21.7 weeks RO DBT, $35 \%$ of all participants were in full remission, and an additional $55 \%$ were in partial remission. A multiple baseline single-case experimental designed study conducted across 13 females $(\operatorname{MED}($ initial episode $)=15, \operatorname{MED}($ Duration=10) shows positive results. In the 40 weeks of treatment, therapists primarily focus on ED psychopathology, following by psychological impairment, life quality, social connectedness, and adaptive control strategies, and $8(62 \%)$ of them completed the whole course. Under the principles of the deviation of the community mean of BMI and Ed psychopath, the complete regression rate of 8 cases was $100 \%$ [19]. It implied that RO DBT might contribute much to Anorexia Nervosa sufferers.

Although not yet mature, researchers still work hard on exploring DBT's pertinence and diversity design. We are of high expectation on DBT's potential in use to bring about improvement in AN.

\subsubsection{Application in China}

As the' second wave' and 'third wave' of behaviorism therapy, visions of CBT and DBT are highly valued by professionals. Although both focus on thinking and reflecting behavior, CBT is more about internal thought patterns, while DBT utilizes those thought patterns to build essential skill sets [20]. So far, no agreement on the theoretical background of DBT has been reached unanimously. However, it is consistent that the concepts are associated with behaviorism theory, dialectical philosophy, and bio-social theory. Some researches even found some considerable similarities between DBT and Zen [19]. Thousand's years of traditional culture provide a favorable environment in applying skill sets of DBT; Thus, the given two therapies exhibit greatly applied potentialities in China.

Although the delayed introduction of DBT in China leads to the lack of experimental study, the similar ideological expression on guided concepts suggests a considerable potentiality in applying.

\subsubsection{Dialectical Philosophy-DBT}

In Radically Open DBT (RO-DBT), Aanorexia nervosa is conceptualized as a disorder of over control. With the guidance of dialectical philosophy, DBT 1) Treat all existed disorders of an individual as a whole but not in separation.2) Therapists value the interaction between rational change and acceptance. Similarities are seen in certain Chinese traditional ideas-Confucianism, Buddhism, and Taoism thinking. After thousands of years, a variety of theoretical has merged to develop. Among that, Neo-Confucianism has established status for the large population in China. It emphasizes three fundamental principles 1) Unity of opposite 2) Struggle and movement 3) Universal connection 4) Change and development. On the one hand, the Chinese have been gradually reconciled to advance dialectical ideas of 
numerous modern values; On the other, the Chinese government found it an integrated part of the education of adolescents, and it has been collected in high school textbooks for decades. Considering the social basis and audience basis of Dialectical ideas, DBT is highly expected to apply effectively with less ideological barriers[19].

\subsubsection{Zen Buddhism-DBT}

The basic assumptions of Zen are to allow the existence of everything that happens at the present moment and that accept world works as a whole; accommodate any opinion that differs from our own; embrace every opinion regardless of moral judgment. It seems to be compatible with three interrelating skills of DBT, and they are observation, description, and integration. Always do the' right thing' but not 'effective things' [20]. The thought of combining concepts of Zen and DBT may provide an edge in treating Chinese patients who Zen Buddhism influences.

\subsubsection{Propagation of CBT in China}

CBT is more prevalent than DBT in China. Current experimental data and simulation results can prove the feasibility of DBT on Anorexia Nervosa.Since1998, the German Chinese psychotherapy institute has trained the first generation of professionals for CBT, represented by the 'Chinese-German class'. Since 2009, the CBT cooperation group of Chinese Medical Association, CBT group of Chinese Psychological Association, CBT special committee of Chinese Mental Health Association and CBT working group of Chinese Medical Association, which also goes to "China CBT professional organization', has been established successively [21]. Recognized by the international community, CBT professional organizations greatly promote CBT's application in China.

\subsubsection{Domestic Experiment-CBT}

From Jan.2008 to Jan.2011, selected 32 women of a Chinese hospital were randomly separated into research groups and control groups. Score variation of HAMD, along with medication follow-up (Recurrence rate in one year), was the primary standard of treatment compliance. It suggested that MiDanPing CBT had considerable effects on improving body weight, decreasing depression problems; increasing treatment compliance, and reducing the recurrence rate of Anorexia nervosa [22].

Table 1. The weight change of participants after different treatments $(n=16, M \pm S D, k g)$

\begin{tabular}{|c|c|c|c|c|c|}
\hline Groups & Start & $\begin{array}{l}\text { After } 2 \\
\text { weeks }\end{array}$ & $\begin{array}{l}\text { After } 3 \\
\text { weeks }\end{array}$ & $\begin{array}{l}\text { After } 6 \\
\text { weeks }\end{array}$ & $\begin{array}{l}\text { After } 12 \\
\text { weeks }\end{array}$ \\
\hline Research & $34.9 \pm 2$ & $35.1 \pm 3$ & $41.8 \pm 4$ & $48.6 \pm 5$ & $49.9 \pm 4$ \\
\hline
\end{tabular}

\begin{tabular}{llllll}
\hline group & 9 & 8 & 3 & 2 & 9 \\
Control & $34.9 \pm 2$. & $34.2 \pm 3$. & $39.9 \pm 4$. & $44.1 \pm 51$ & $45.0 \pm 5$. \\
group & 8 & 1 & 2 & & 2 \\
Number of & & & & & \\
$P$ & $>0.05$ & $>0.05$ & $>0.05$ & $>0.05$ & $>0.01$ \\
\hline
\end{tabular}

Table 2. Score change in HAMD $(n=16, M \pm S D)$

\begin{tabular}{|c|c|c|c|c|c|}
\hline Groups & Start & $\begin{array}{l}\text { After } 2 \\
\text { weeks }\end{array}$ & $\begin{array}{l}\text { After } 3 \\
\text { weeks }\end{array}$ & $\begin{array}{l}\text { After } 6 \\
\text { weeks }\end{array}$ & $\begin{array}{l}\text { After } 12 \\
\text { weeks }\end{array}$ \\
\hline $\begin{array}{l}\text { Research } \\
\text { group }\end{array}$ & $\begin{array}{l}30.2 \pm 4 \\
9\end{array}$ & $\begin{array}{l}27.2 \pm 4 . \\
6\end{array}$ & $\begin{array}{l}21.2 \pm 4 . \\
3\end{array}$ & $\begin{array}{l}13.3 \pm 1 . \\
5\end{array}$ & $6.3 \pm 3.3$ \\
\hline $\begin{array}{l}\text { Control } \\
\text { group }\end{array}$ & $\begin{array}{l}30.0 \pm 4 \\
8\end{array}$ & $\begin{array}{l}26.9 \pm 4 . \\
7\end{array}$ & $\begin{array}{l}21.4 \pm 4 . \\
2\end{array}$ & $\begin{array}{l}36.2 \pm 4 . \\
6\end{array}$ & $\begin{array}{l}10.6 \pm 3 . \\
7\end{array}$ \\
\hline $\begin{array}{l}\text { Number of } \\
\text { P2 }\end{array}$ & $>0.05$ & $>0.05$ & $>0.05$ & $>0.05$ & $>0.01$ \\
\hline
\end{tabular}

Table 3. Comparison on compliance [n(\%)]

\begin{tabular}{|c|c|c|c|c|c|c|}
\hline & $\mathrm{n}$ & $\begin{array}{l}\text { Passive } \\
\text { medication }\end{array}$ & $\begin{array}{l}\text { Active } \\
\text { medicati } \\
\text { on }\end{array}$ & $\begin{array}{l}\text { Refuse to } \\
\text { take } \\
\text { medicatio } \\
\mathrm{n}(\mathrm{a})\end{array}$ & $\begin{array}{l}\text { With } \\
\text { referral } \\
\text { groups } \\
\text { (b) }\end{array}$ & $\begin{array}{l}\text { Without } \\
\text { referral } \\
\text { groups }\end{array}$ \\
\hline $\begin{array}{l}\text { Research } \\
\text { group }\end{array}$ & 16 & $\begin{array}{r}2 \\
(12.5)\end{array}$ & $\begin{array}{l}4 \\
(25.0)\end{array}$ & $\begin{array}{l}10 \\
(62.5)\end{array}$ & $\begin{array}{l}10 \\
(62.5)\end{array}$ & $\begin{array}{l}6 \\
(37.5)\end{array}$ \\
\hline $\begin{array}{l}\text { Control } \\
\text { group }\end{array}$ & 16 & $\begin{array}{r}5 \\
(31.2)\end{array}$ & $\begin{array}{r}6 \\
(37.5)\end{array}$ & $\begin{array}{l}5 \\
(31.5)\end{array}$ & $\begin{array}{c}7 \\
(43.8)\end{array}$ & $\begin{array}{r}9 \\
(56.2)\end{array}$ \\
\hline
\end{tabular}

\section{LIMITATION AND FUTURE DIRECTION}

Firstly, even though it is widely accepted that CBT turns out to be an effective treatment for Anorexia Nervosa, Ball and Mitchell (2010) found no effect of CBT in treating mild depression in patients diagnosed with anorexia nervous, while depression is obviously one of the main commodities of Anorexia. We need more in-depth research on Anorexia Nervosa samples and the effectiveness of CBT, as literature in treating anorexia nervous with CBT is yet too sparse to find. Also, according to Bakes (2014), few randomized, controlled studies evaluating various general treatments for patients with anorexia nervous have been presented so far. High attrition rates and pharmaceutical confounds are often limiting factors in studying populations diagnosed with anorexia nervous, and they must be considered [4].

Since CBT works through change, adjustment, or improving the patient's incorrect body image, cultural context is undoubtedly crucial to think about. The counselor needs to do a lot of research to get an approach to or better understand the etiology of the 
patient under his/her corresponding culture. Certain behaviors or ideas that turn out to be correct or proper in culture might be improper in another culture. In addition, the counselor will not be capable of offering the most effective treatment or therapy unless he or she knows why this patient is thinking this way based on their culture and identity. When CBT is applied to the patient, I think expressive writing therapy also works well, helping the patient describe or express what they feel about the incident or say about their figures in this case.

Secondly, most of the available research on the efficiency of DBT covered the small scope of samples with a specific identity, most especially, there is no experimental evidence can confirm its effectiveness in China Thus, more research and experiment are suggested to be done for determining how much potential DBT would have as for promising psychological treatment of Anorexia Nervosa. Besides, the irreconcilable clash on culture, region, belief, race, and class should be considered when optimizing the previous design in the principles of DBT for greater influence and possibility of acceptance in China.

\section{CONCLUSION}

Current studies on empirical evidence associate with theory support have proven the considerable feasibility of CBT and DBT to Chinese adolescents who already suffered from Anorexia Nervosa. Although the narrow propagation eating disorder added up the difficulties of approaching new therapeutic techniques for those people in need, these two therapies will be of great potentiality for being widely applied in china once the theories get better promotion. They provide a new perspective for psychological treatment after being combined with China's traditional cultural factors. Basing on current research, it is believed to substantially reduce the high prevalence and improve cure efficiency of Anorexia Nervosa in a future application.

Asian appreciation of slim body adds up Chinese adolescent's anxiety about body image. Without proper guidance, some impressionable adolescents choose to lose weight irrationally to meet their inside need of seeking an identity in a society dominated by "perfect image". Meanwhile, modern forms of parental involvement are a traditional feature of Chinese culture. The traditional preference for obedient kids and high expectations conveyed to kids also made those youths that are easily dominated by negative emotions. It may be a potential factor, which exposes adolescents to over-control on weight. What's worse? Chinese are commonly void of knowledge of Anorexia Nervosa and other relative eating disorders. People don't realize the gravity of its seriousness, which makes it a great barrier to the application of CBT\&DBT on those targeted Anorexia Nervosa patients with mental issues.

\section{REFERENCES}

[1] American Psychiatric Association. (2013). Anxiety disorders. In Diagnostic and statistical manual of mental disorders (5th ed.). DOI: https://doi.org/10.1176/appi.books.9780890425596 .dsm05

[2] Yvette Brazier in: Anorexia nervosa: What you need to know, 2021, DOI: https://www.medicalnewstoday.com/articles/26743 2

[3] Sartorius N, Holt RIG, Maj M (eds): Comorbidity of Mental and Physical Disorders. Key Issues Ment Health. Basel, Karger, 2015, vol 179, pp 66-80 DOI: https://doi.org/10.1159/00036553

[4] Oakes, Brittany, Efficacy of Cognitive Behavioral Therapy in Treating Anorexia Nervosa: A Review of Literature, UC Merced Undergraduate Research Journal, $\quad 7(2), \quad 2014, \quad$ DOI: https://escholarship.org/uc/item/2c06j71p

[5] Mayo Clinic staff in: Risk factors and complications of ED, 2018. Available at: https://www.mayoclinic.org/diseases-conditions/ea ting-disorders/symptoms-causes/syc-20353603\#: :t ext $=$ The $\% 20$ exact $\% 20$ cause $\% 20$ of $\% 20$ eating $\% 20 \mathrm{~d}$ isorders $\% 20$ is $\% 20$ unknown.,chemicals $\% 2 \mathrm{C} \% 20 \mathrm{~m}$ ay\%20play\%20a\%20role\%20in\%20eating\%20diso rders.

[6] Laura Angers in: Common Causes Of Eating Disorders, 2020. Available at: https://www.betterhelp.com/advice/eating-disorder s/common-causes-of-eating-disorders/?utm_source $=$ AdWords\&utm_medium $=$ Search_PPC_c\&utm_te rm=_b\&utm_content $=118051370287 \&$ network $=\mathrm{g}$ $\&$ placement $=\&$ target $=\&$ matchtype $=b \& u t m \_$campa ign $=11771068538 \&$ ad_type $=$ text $\&$ adposition $=\& g c$ lid=CjwKCAjwlrqHBhByEiwAnLmYUEhAsJ4BE 9t2JQVLrTrQW0rBnp4TRo7xuf0Qp0opj9WH5B MfxmdNnRoCIeEQAvD_BwE

[7] Krisna Patel, Kate Tchanturia, and Amy Harrison, in: An Exploration of Social Functioning in Young People with Eating Disorders: A Qualitative Study, PLoS One. 2016; 11(7): e0159910. Published online 2016 Jul $26 . \quad$ DOI: 10.1371/journal.pone.0159910, https://www.ncbi.nlm.nih.gov/pmc/articles/PMC49 61427/

[8] The Emily Program , How Do Eating Disorders Affect Relationships? Published in 2020. DOI: https://www.emilyprogram.com/blog/how-do-eatin g-disorders-affect-relationships/ 
[9] Koji TSUBOI, in: Eating Disorders in Adolescence and Their Implications, JMAJ 48(3): 123-129, 2005,

DOI:https://www.med.or.jp/english/pdf/2005_03/1 23_129.pdf

[10] Society of Clinical Psychology, in: COGNITIVE BEHAVIORAL THERAPY FOR ANOREXIA NERVOSA, DOI: https://div12.org/treatment/cognitive-behavioral-th erapy-for-anorexia-nervosa/

[11] Carter, J. C., McFarlane, T. L., Bewell, C., Olmsted, M. P., Woodside, D. B., Kaplan, A. S., \&

[12] Editorial Staff, What Are the Techniques Used in DBT(Dialectical Behavior Therapy)? Published in 2020.

DOI:https://recoveryfirst.org/dialectical-behavior-t herapy/?_cf_chl_jschl_tk__=pmd_5995e2700d32 687e1e58f35d2cde6748a36e4934-1628579906-0-g qNtZGzNAjijcnBszQe6

[13] Mirror-Mirror, DBT in the Treatment of Eating Disorders, 2021.2 DOI: https://mirror-mirror.org/getting-help/dbt-in-the-tre atment-of-eating-disorders

[14] Behavioral Tech,How DBT Can Help Treat Eating Disorders, 2016.2 DOI: https://behavioraltech.org/dbt-treat-eating-disorders /

[15] Mauirecovery, Understanding The Difference Between CBT and DBT Therapy, 2021. DOI:https://mauirecovery.com/cbt-and-dfbt-therap $\mathrm{y} /$ understanding-the-difference-between-cbt-and-d bt-therapy/

[16] Lucene Wisniewski, Ph.D., Denise D Ben-Porath, Ph.D.Dialectical Behavior Therapy and Eating Disorders: The Use of Contingency Management Procedures to Manage Dialectical Dilemmas, 2018. DOI:https://doi.org/10.1176/appi.psychotherapy.20 15.69.2.129

[17] [Julianna Gorder, Radically Open Dialectical Behavior Therapy (RO DBT) for Anorexia Nervosa: Clinical Applications in a Nutshell, 2019. DOI:https://www.edcatalogue.com/radically-opendialectical-behavior-therapy-ro-dbt-anorexia-nervo sa-clinical-applications-nutshell/

[18] Thomas R. Lynch, Roelie J. Hempel, Signaling Matters: Radically Open-Dialectical Behavior Therapy for Anorexia Nervosa, Proceedings of the Springer, Singapore, 2017.

[19] Tan Mengling, Ren Zhihong, Zhao Chuanxiao, Jiang Guangrong, Dialectical Behavior Therapy:
Theoreticcal Background, Efficacy, and Mechanism, Processing of Journal of Psychological Science, Vol.44, No.2, pp.481-488, 2021.

[20] Perround, N., Nicastro, R., Jermann, F.,\&Huguelet,P.Mindfulness skills in Borderline personality disorder patients during dialectical behavior therapy: preliminary results. Processing by Psychiatry in Clinical practice, 16(3). 189-196.

[21] CBT China, What is CBT, 2021. DOI: http://www.cbtchina.com.cn

[22] Wang, Erfang, Li Jianmin, Song Xiaohong, Zhang Yutao, Efficacy of Mirtazapine Combined with cognitive behavior in the treatment of anorexia nervosa. Processing of International Medicine\& Health Guidance News, Vol.4, pp.527-529, 202 